\title{
Using Genetic Algorithm to Reduce the Noise Effect on Images \\ Baydaa S Bhnam
}

Baydaa_sulaiman@uomosul.edi.iq

College of Computer Science and Mathematics

University of Mosul

\section{Received on: $25 / 10 / 2011$}

\section{ABSTRACT}

Accepted on : 14/12/2011

This paper deals with a problem that concentrates on the noise removal that the images are affected from different resources employing Genetic Algorithm with filters. To achieve the aims of the paper, six types of genetic filters are suggested for noise removal from the images. These suggested genetic filters depending on filters (mean, median, min and max) as an objective function for them.

These suggested genetic filters are applied on several real images contaminated by two types of noise with different levels for comparison and to show the effectiveness of them. The result show that The fifth genetic filter that depends on the median filter as an objective function and heuristic crossover and adding and subtracting mutation, gives the best results with $\mathrm{RMSE}=15.7243$ and $\mathrm{PSNR}=24.1646$ for Lena.bmp image and with RMSE=8.6197 and PSNR=29.4210 for girl.png image when add 0.05 salt \& paper noise.

Keywords: Genetic Algorithm, Image Enhancement, Filters , Noise , Digital Image .

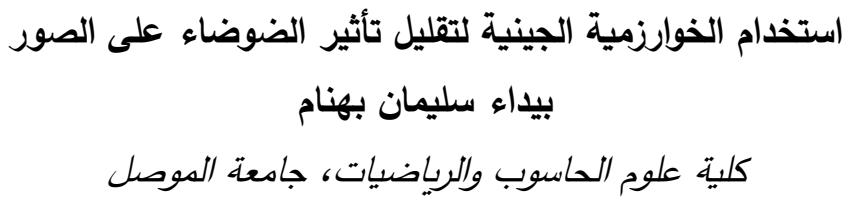

\section{الملخص}

تتتاول الورقة البحثية مشكلة إزالة الضوضـاء الذي تتعرض لها الصورة من عدة مصـادر وذلك باستخدام الخوارزمية الجينية (Genetic Algorithm) مع المرشحات. ولتحقيق أهداف البحث تم اقتراح ستة مرشحات جينية لإزالة الضوضاء من الصور • واعتمدت هذه المرشحات الجينية المقترحة على المرشحات المعروفة (المعدل، الوسيط، التصغير والتكبير) بوصفها دالات هدف لها.

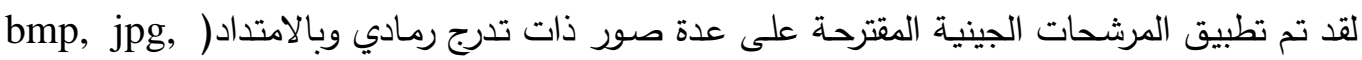

(png ) شوهت بنوعين من الضوضاء وبنسب مختلفة وتم إجراء مقارنة بين هذه المرشحات لمعرفة أكفأها. وتبين أن المرشح الجيني الخامس الذي اعتمد على دالة الهدف مرشح الوسيط وعملية التداخل الابدالي

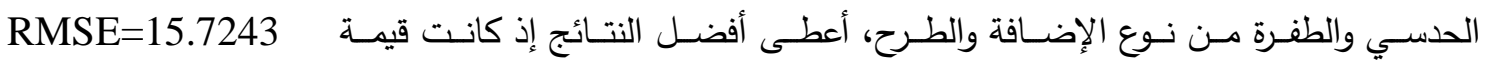

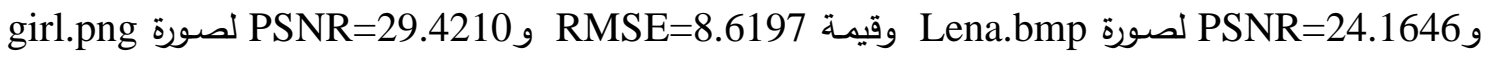

$$
\text { عند إضافة ضوضاء نوع حبات الملح والفلفل بنسبة 0.05. }
$$

الكلمات المفتاحية: الخوارزمية الجينية ، تحسين الصورة ، المرشحات ، الضوضاء ، الصورة الرقمية.

$$
\text { 1. 1 المقدمة ومنهجية البحث }
$$

إن ما يؤشره الواقع الحالي من التطور الهائل في العديد من المجالات وعدم قدرة الطرائق التقليدية بإيجاد حلول ملائمة، يؤشر وبدون شك الخطوة الضرورية باتجاه التوصل إلى حلول تسهم في الارتقاء بكفاءة. لذا فقد تم 
إيجاد خوارزميات كفوهة تساعد في إيجاد الحلول المثالية للمسائل المعقدة وبطرق ذكائية. ومن هذه الخوارزميات الكفوءة الخوارزميات الجينية[1]. اكتثف الخوارزمية الجينية عام 1975 العالم جون هولاند John Holland في جامعة ميشكان، فقد نشر

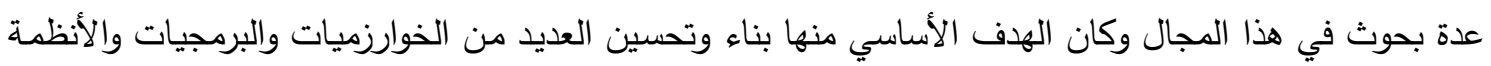
باستخدام هذه الخوارزمية[3]. تتضمن الخوارزمية الجينية عددا من الخطوات الأساسية، هذه الخطوات ثابتة لمختلف المسائل ولكل التطبيقات ويكون الاختلاف في أسلوب صياغة كل خطوة من هذه الخطوات وتطبيقها حسب المسالة. إن خطوات

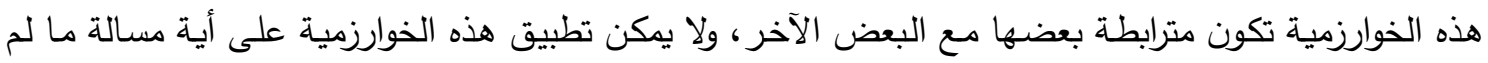

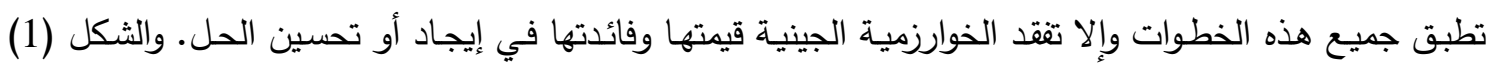
يوضح المخطط العام للخوارزمية الجينية[1]:

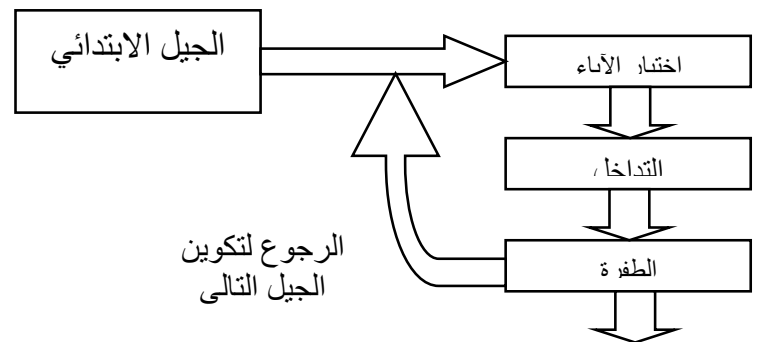

\section{الثكل (1): يوضح المخطط العام للخوارزمية الجينية}

وقد استخدمت الخوارزمية الجينية بثكل واسع في العديد من المجالات منها: معالجة الصور ( Image

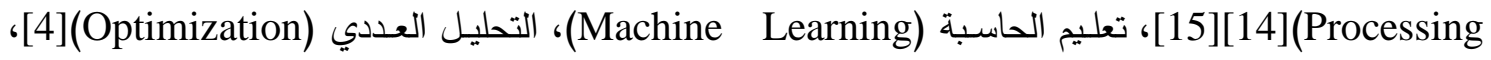
بحوث العمليات (Operations Research)، وحل مسائل التشفير وكسر الثفرة (Cipher \& Decipher) وغيرها من المجالات التي لاقت بها نجاحا كبيرا [3].

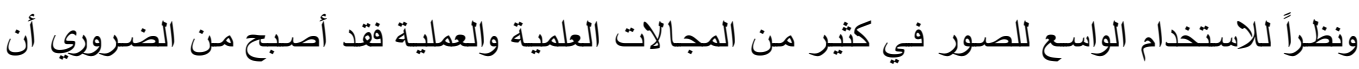
تكون الصور واضحة وتقضي الغرض الذي أخذت لأجلهِ[2][16]، لذلك شهَّ مجال التعامل مـع الصور الرقمية

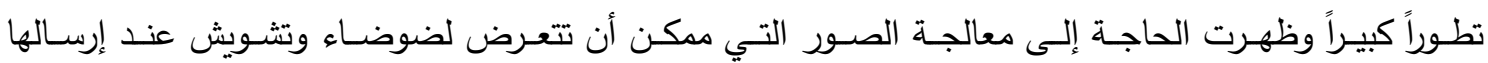
واستقبالها][6[13][20]. وان الضوضاء هي عن عبارة عن معلومات غير مرغوب فيها وغير مفيدة تسبب تشويهاً

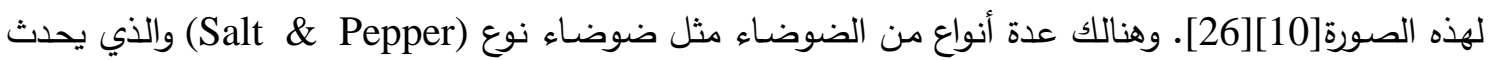

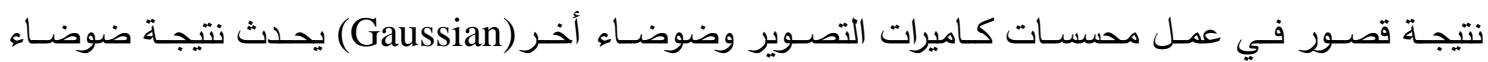
الكتروني[7] · وهذان النوعان تم استخدامهما في البحث.

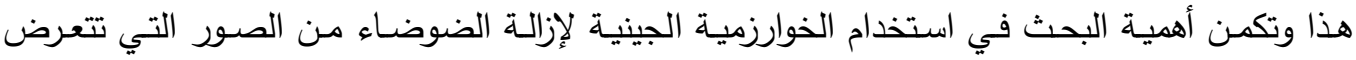
لضوضاء وتثويش عند إرسالها واستقبالها لغرض الحصول على صورة تطابق إلى حد كبير الصورة الأصلية. لذا

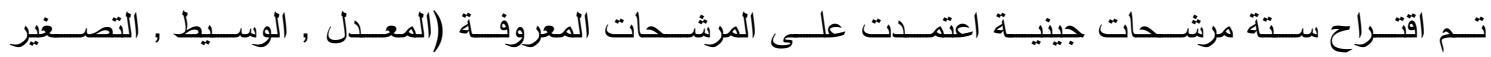
والتكبير)][5][17][19][24] كدالات هدف لها. ولأجل مقارنـة الأداء لكل من هذه المرشحات المقترحة فقد تم حساب نسبة الخطأ (RMSE) قبل إضافة الضوضـاء للصورة وبعد معالجتها بالمرشح الجيني المقترح. وكذلك تم 
حساب PSNR(Peak Signal to Noise Ratio) الذي يقيس مدى تطابق الصور الأصلية مع الصور

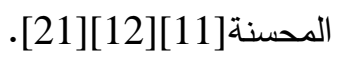

تثكل الدراسات التطبيقية السابقة أهمية بالغة عند إعداد هذا البحث ومن هذه الدراسات: 1. فقد تتاولت دراسة (Van De Ville) مرشح المعدل ومرشح adaptive wiener المهجنين مع المنطق

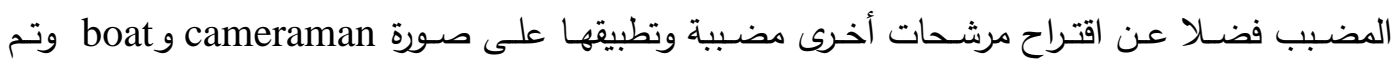

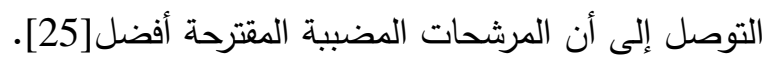
2. تتاولت دراسة (How-Lung E. and Kai-Kuang M) اقتراح اعتمـادا على مرشـح الوسيط وتطبيقه على صور متعددة ومقاطع فيديويـة وأعطى المرشح المقترح نتائج أفضل من بقية التقنيات المستخدمة بالبحث] [9].

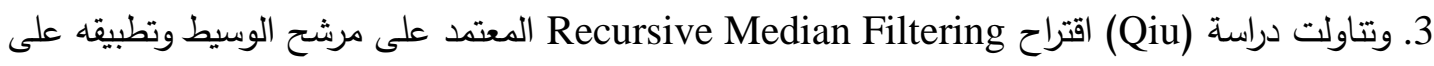

صورة lena وقد حصل على نسبة خطا 35.1 [18]. 4. وكانت دراسة (Gupta, G.) عن اقتراح طريقة لتحسين مرشح الوسيط وإجراء مقارنة بين الطريقة المحسنة

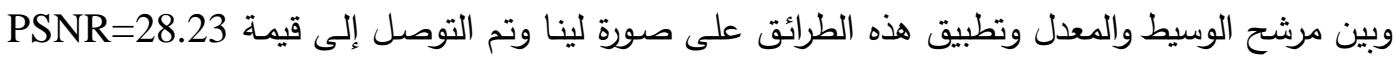

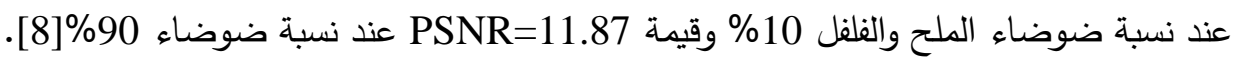
5. اقتراح Siltering using (Sovilj-Nikic, S. and Sovilj-Nikic, I.) تتاولت دراسة partition based median filter (PBM) التوصل إلى SNR(db)=27.71 لصورة lena المشوشة بضوضاء كاوس[22]. 2. تعتمد خوارزميات المرشحات الجينية المقترحة على حسـاب دالـة الهـف اعتمـادا على مرشـح المعدل، الوسيط والتكبير والتصغير • وفيما يأتي طريقة كل مرشح جيني مقترح: 1.2

يعتمد هذا المرشح على مرشح المعدل بحساب دالة الهدف لعناصر نافذة من الصسورة، وخطوات هذا المرشح هي: الخطوة الأولى: قراءة الصورة الأصلية، ثم يتم إضافة ضوضاء عليها بنسبة معينة. الخطوة الثانية: اخذ نافذة من الصورة بأبعاد معينة ولتكن 3*3. الخطوة الثالثة: حساب دالة الهدف F لعناصر النافذة باستخدام مرشح المعدل كما في المعادلة (1) [5][1(17]: $F=\operatorname{mean}(P(:))$

$$
\text { حيث أن P تمثل النافذة التي تتم معالجتها. }
$$

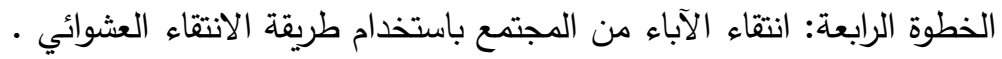

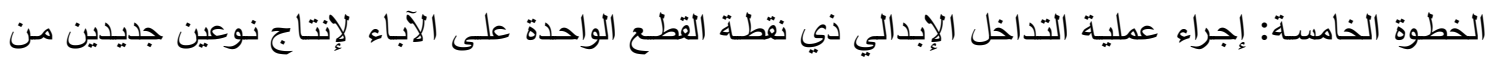
الأبناء. الخطوة السادسة: تطبيق الطفرة من نوع تغيير الرتب. 
الخطوة السابعة: حساب نسبة الخطأ RMSE للنافذة الناتجة. ويتم التوقف إذا تم الوصول إلى عدد الأجيال المحدد وإلا الرجوع إلى الخطوة الثالثة.

الخطوة الثامنة: انتقاء النافذة الأقل نسبة خطأ ووضعها في مصفوفة B. ثم الرجوع إلى الخطوة الثانية ويتم التوقف لحين قراءة جميع نوافذ الصورة. والثكل (2) يمثل المخطط الانسيابي للمرشح الجيني الأول. البداية
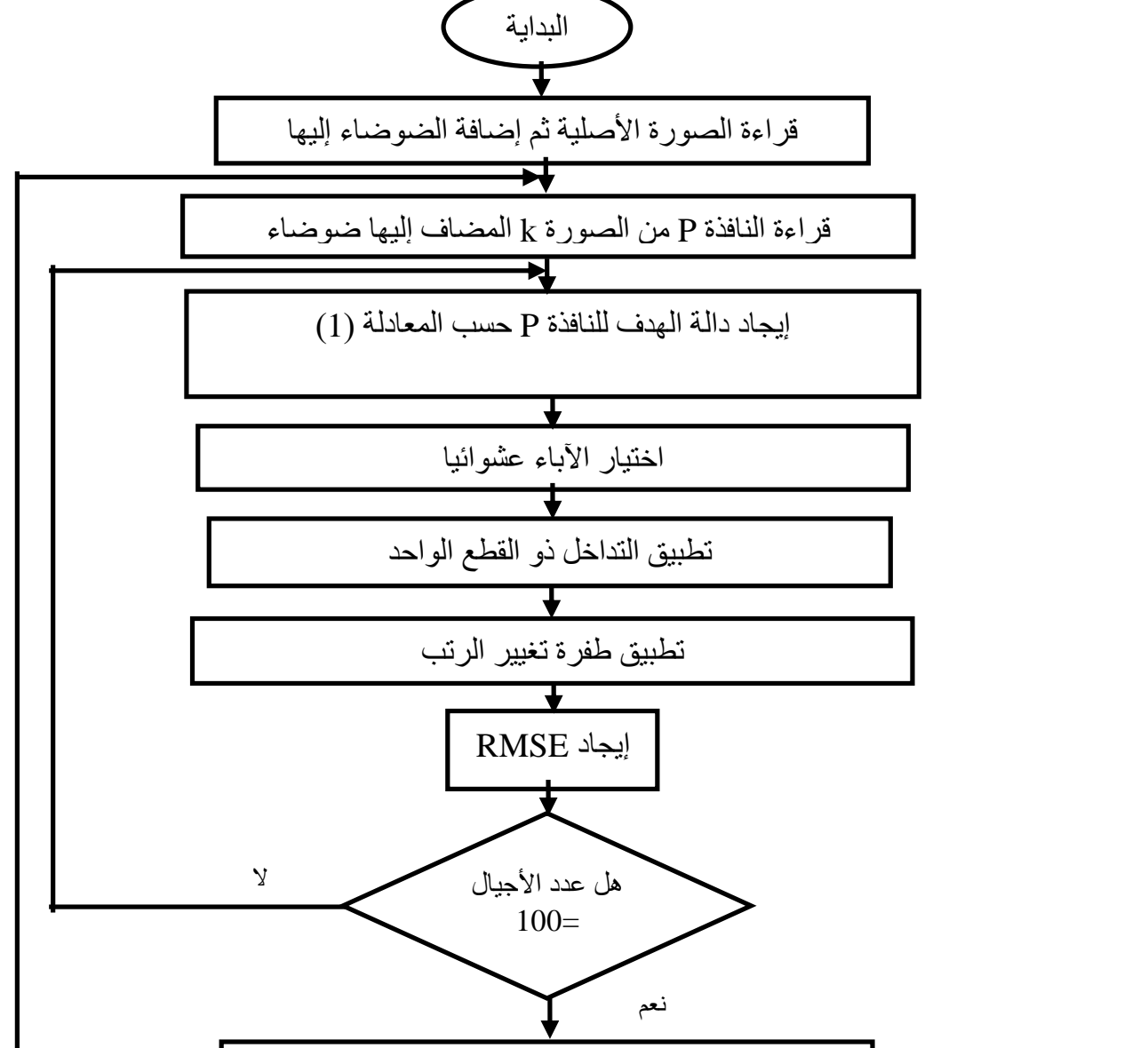

اختبار النافذة الأقل RMSE ووضعها في مصفوفة B y

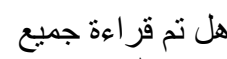

نو افذ الصورة أعرة نمع

إيجاد RMSE و RSNR للصورة B

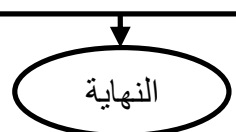

الثكل(2): المخطط الانسيابي للمرشح الجيني الأول 
وان المثال التالي يوضح المرشح الجيني الأول: بعد قراءة الصورة الأصلية وإضافة الضوضاء إليها، سيتم قطع نافذة بحجم معين وليكن 3*3 من الصورة الصناء K المضاف إليها الضوضاء فثثلا كانت النافذة كالآتي:

\begin{tabular}{|c|c|c|}
\hline 180 & 167 & 165 \\
\hline 171 & 170 & 158 \\
\hline 167 & 0 & 148 \\
\hline
\end{tabular}

نحسب دالة الهدف لهذه النافذة باستخدام مرشح المعدل وحسب المعادلة (1) وبهذا تكون قيمة 147= F= ثم نزاوج كل نقطتين في النافذة باستخدام طريقة التداخل ذي النقطة الواحدة لإنتاج نقطتين جديدتين فمثلا نزاوج النقطتين: 180 و 167 كالآتي:

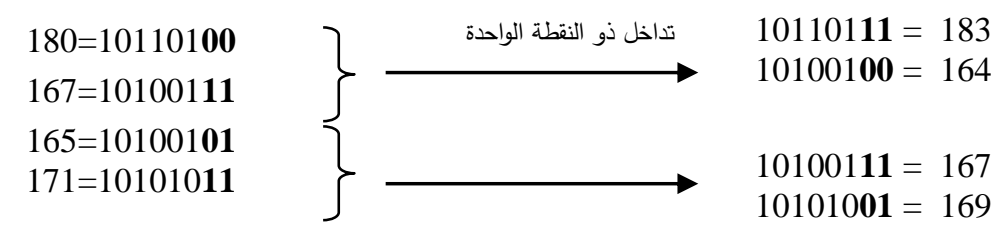

فمثلا النقطة 10100100=164 تصبح بعد تطبيق الطفرة 10100001=161 وهكذا بالنسبة لبقية نقاط

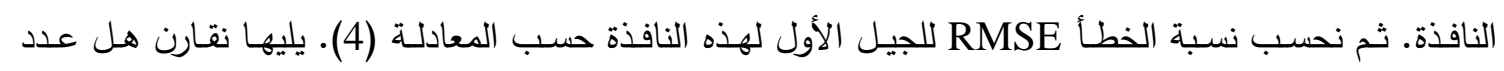

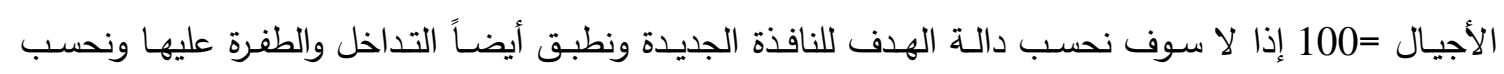

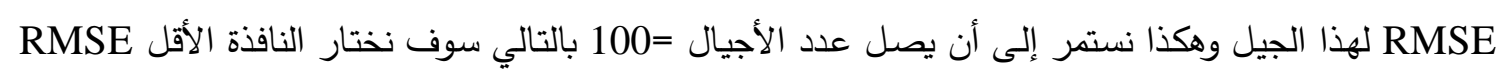

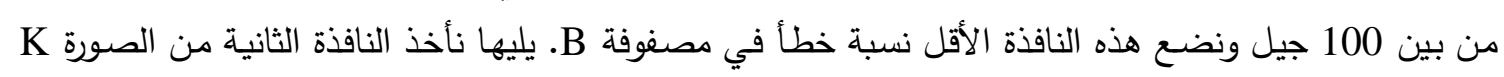

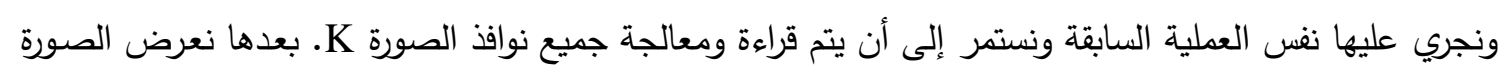
B ونحسب 2.2 المرشح الجيني الثاني

يعتمد هذا المرشح على مرشح الوسيط لحساب دالة الهدف لعناصر نافذة من الصورة.وان خطوات هذا

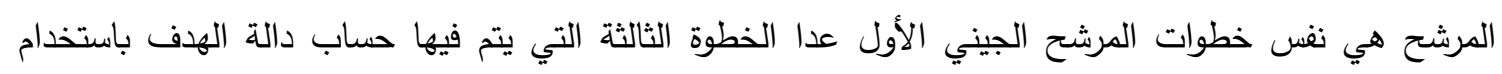

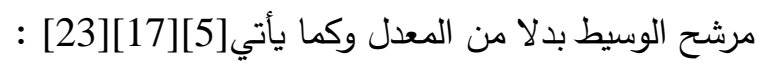

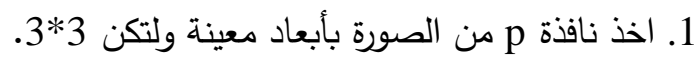

2. ترتيب نقاط النافذة ترتيبا تصاعديا. $F=\operatorname{median}(P(:))$

3. حساب دالة الهدف F لعناصر النافذة باستخدام مرشح الوسيط كما في المعادلة (2):

3.2 المرشح الجيني الثالث

في هذا المرشح يتم حساب دالة الهدف باستخدام مرشح التصغير والتكبير لذا فان التغيير أيضاً في

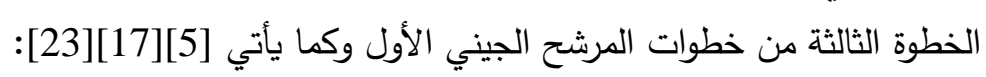

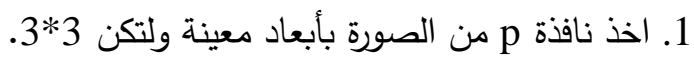

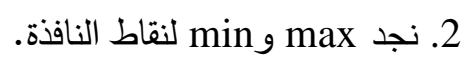


3. حساب دالة الهدف F لعناصر النافذة باستخدام مرشح التصغير والتكبير كما في المعادلة (3):

$F=\max (P(:)) \quad$ if $P(i, j)>\max$

$F=\min (P(:)) \quad$ if $P(i, j)<\min$

$F=P(i, j) \quad$ if $\min \leq P(i, j) \leq \max$

4.2

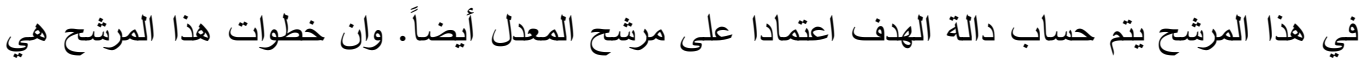

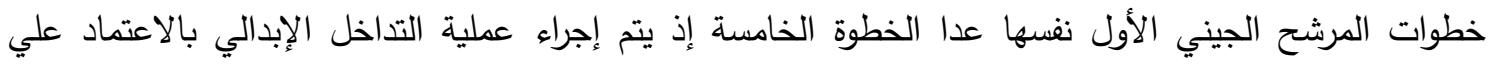
الحدس بقيمة Ratio مساوية لـ 0.1، وكذلك الخطوة السادة فقد تم تطبيق الطفرة من نوع الإضافة والطرح بمقدار

5.2

إن خطوات هذا المرشح هي نفس خطوات المرشح الجيني الرابع عدا انه تم حساب دالة الهـف اعتمادا على مرشح الوسيط.

6.2

إن خطوات هذا المرشح هي نفس خطوات المرشح الجيني الرابع عدا انه تم حساب دالة الهـف اعتمادا على

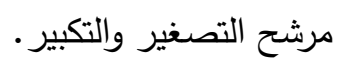

3. قياس أداء تطبيق المرشحات الجينية المقترحة

لغرض قياس أداء تطبيق المرشحات الجينية المقترحة فقد تم تطبيقها على أنواع مختلفة من الصور بنافذة

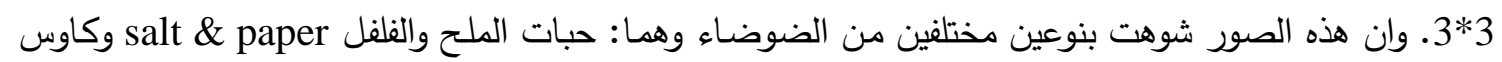
Gaussian معالجتها بالمرشحات الجينية المقترحة كما في المعادلة (4) [11][12][12]: $R M S E=\sqrt{\frac{1}{M * N} \sum_{r=0}^{M-1} \sum_{c=0}^{N-1}\left[\operatorname{Im}_{\text {new }}(r, c)-\operatorname{Im}_{\text {old }}(r, c)\right]^{2}}$

وكذلك تم حسـاب PSNR)-Peak Signal-to-noise ratio الذي يوضـح مدى تطابق الصـور

الأصلية مع الصور المحسنة كما في المعادلة (5)[121][12] :

$P S N R=10 \log 10(L-1)^{\wedge} 2 / \frac{1}{M * N} \sum_{r=0}^{M-1} \sum_{c=0}^{N-1}\left[\operatorname{Im}_{\text {new }}(r, c)-\operatorname{Im}_{\text {old }}(r, c)\right]^{2}$

حيث أن L أكبر تدرج رمادي في الصورة المعالجة. 


\section{4. نتائج تطبيق المرشحات الجينية المقترحة}

لقد تم تطبيق الخوارزميات الجينية المقترحة على عدة صور مشوهة بنوعين مختلفين من الضوضـاء وبنسب

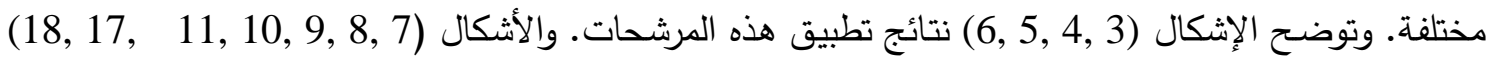
16, 15, 14, 13, 12 , الصور المسترجعة بالمرشحات الجينية المقترحة.
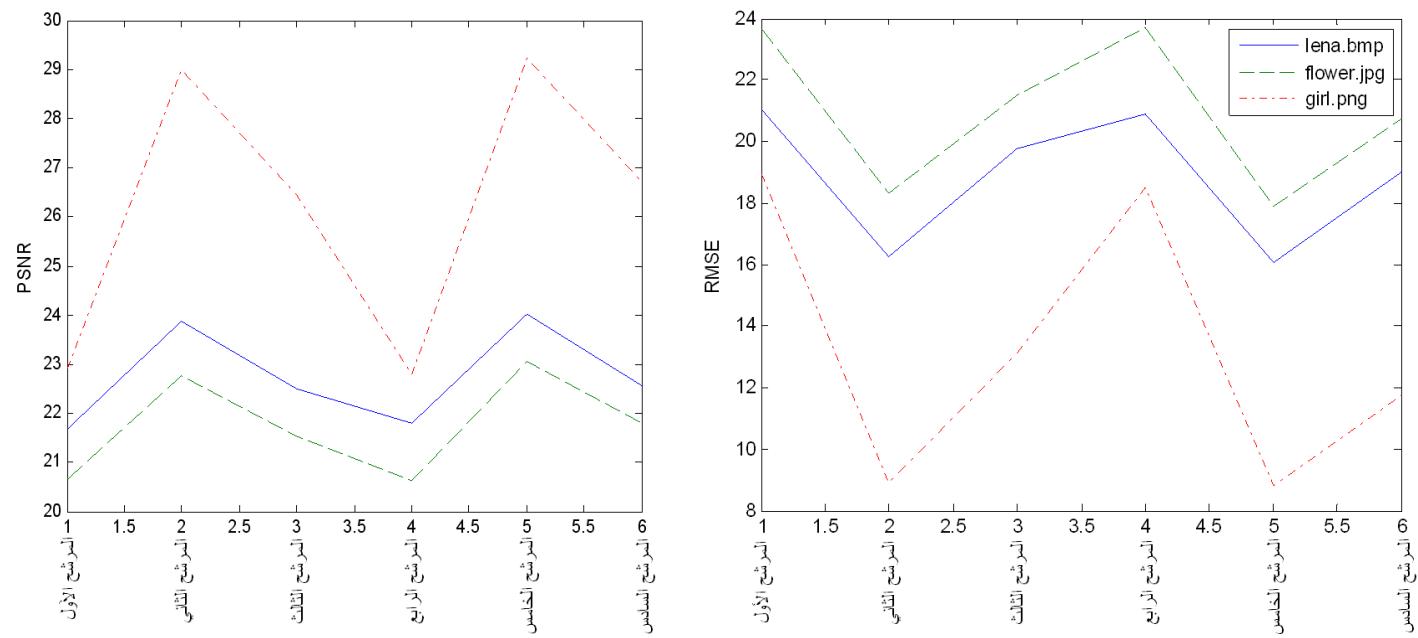

الثكل (3): نسبة الخطأ(RMSE) و (PSNR) عند إضافة ضوضاء نوع حبات الملح والفلفل بنسبة 0.1
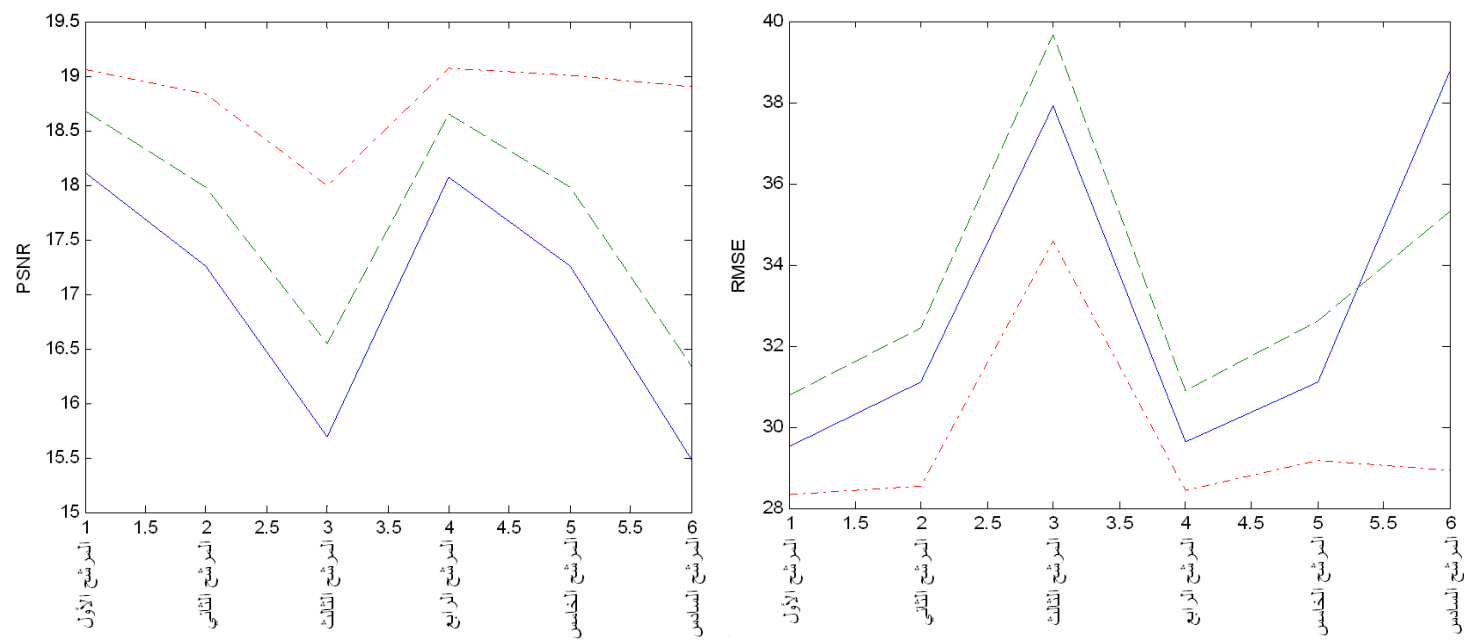

الثكل (4): نسبة الخطأ(RMSE) و) (PSNR) عند إضافة ضوضاء نوع كاوس بنسبة 0.1 

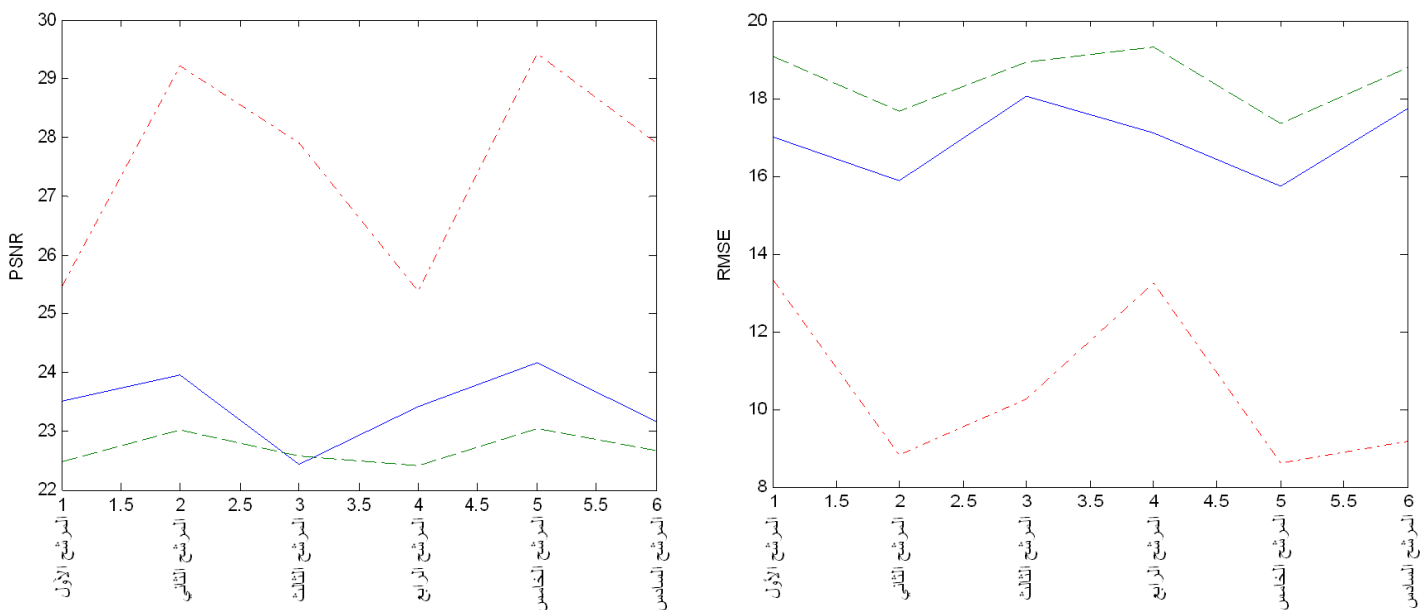

الثكل (5): نسبة الخطأ(RMSE) و) (PSNR) عند إضافة ضوضاء نوع حبات الملح والفلفل بنسبة 0.05
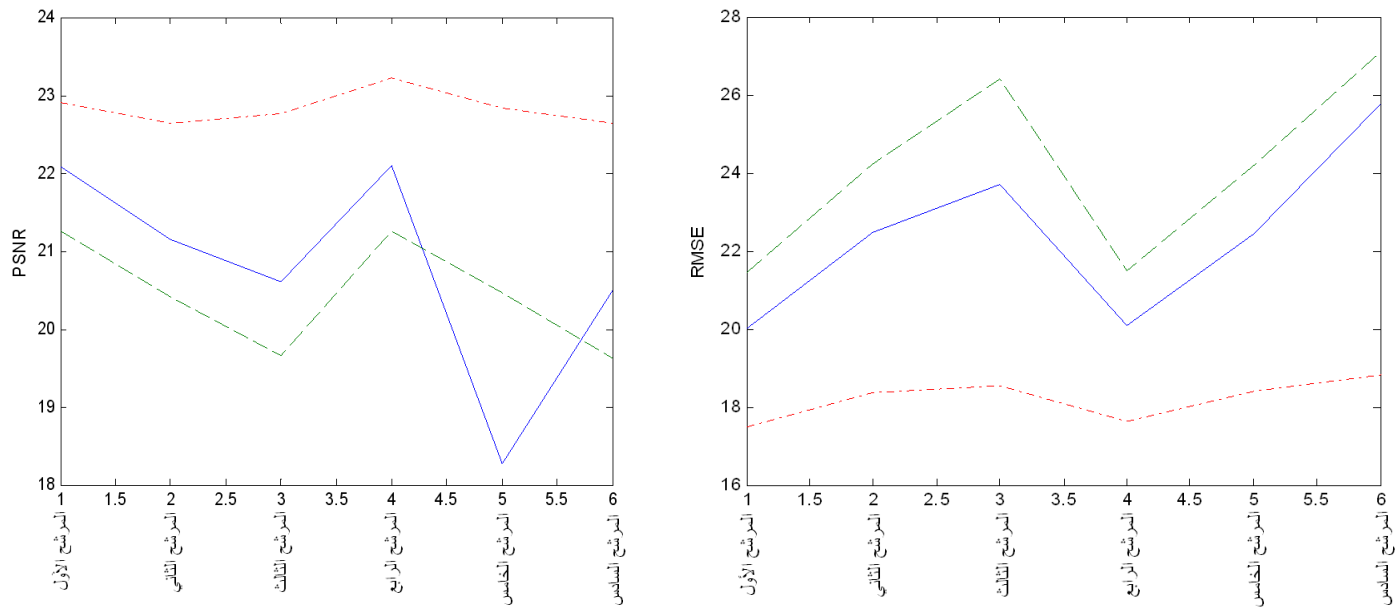

الثكل (6): نسبة الخطأ(PSNR) و (PSN) عند إضافة ضوضاء نوع كاوس بنسبة 0.05

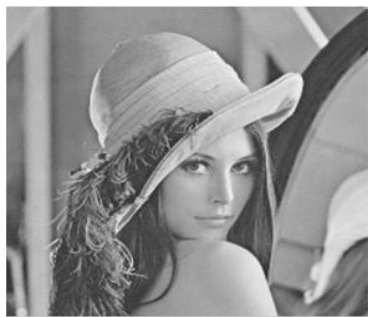

a

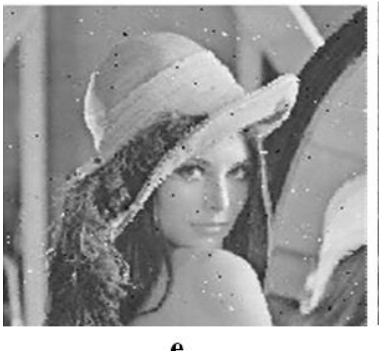

e

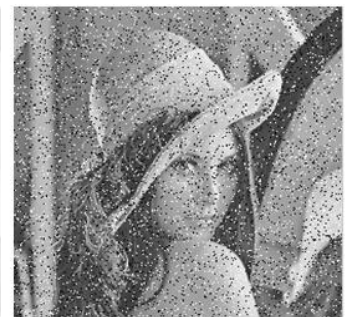

b

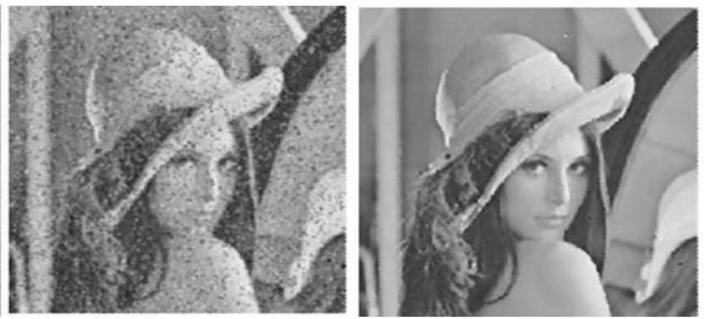

c

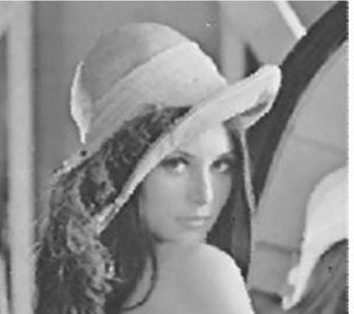

$\mathbf{g}$

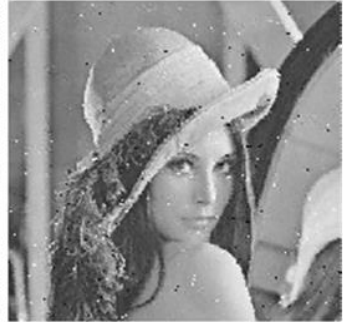

$\mathbf{h}$

الثكل (7): نتائج المرشحات الجينية على صورة Lena والضوضاء نوع حبات الملح والفلفل بنسبة 0.1 (a) الصورة الأصلية. (b) الصورة المشوهة. (c) الصورة المسترجعة بالمشح الجيني الأول. (d) الصورة المسترجعة بالمرشح الجيني

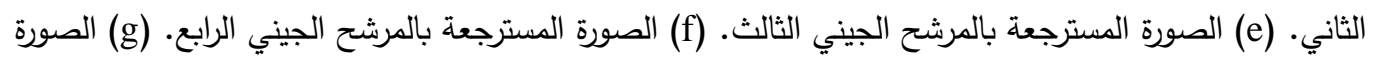
المسترجعة بالمرشح الجيني الخامس. (h) الصورة المسترجعة بالمشح الجيني الساد. 

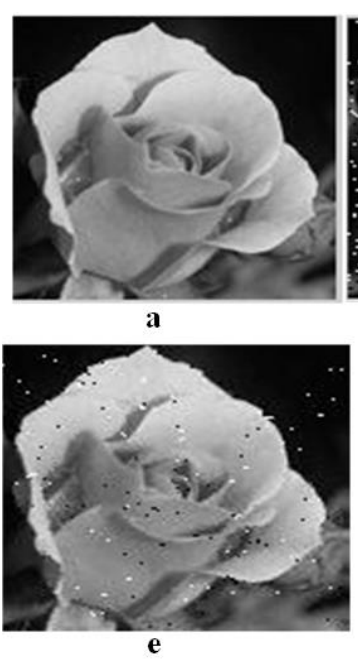

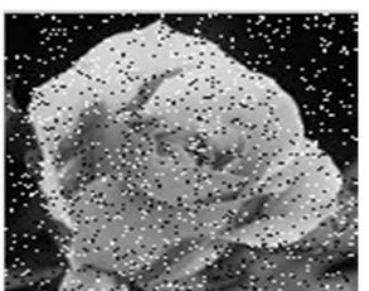

b

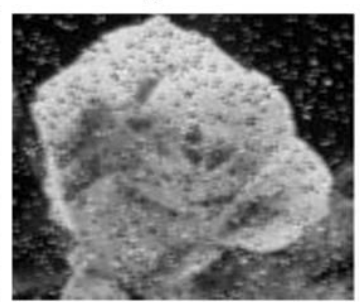

f

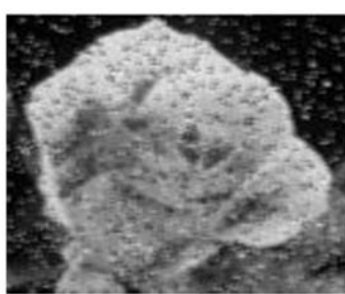

c

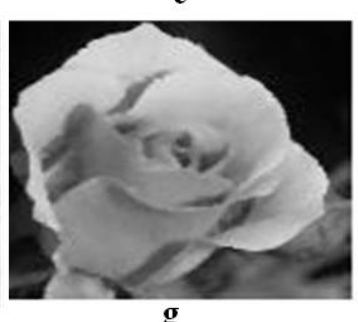

g

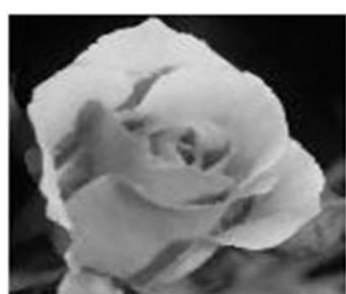

d

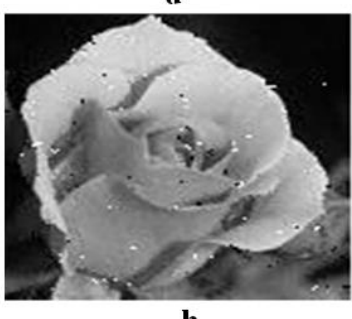

h

الثكل(8): نتائج المرشحات الجينية على صورة Flower والضوضاء نوع حبات الملح والفلفل بنسبة 0.1. (a) الصورة الأصلية. (b) الصورة المشوهة. (c) الصورة المسترجعة بالمرشح الجيني الأول. (d) الصورة المسترجعة بالمرشح الجيني

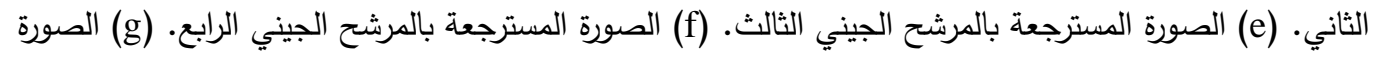
المسترجعة بالمرشح الجيني الخامس. (h) الصورة المسترجعة بالمرشح الجيني السادئ.
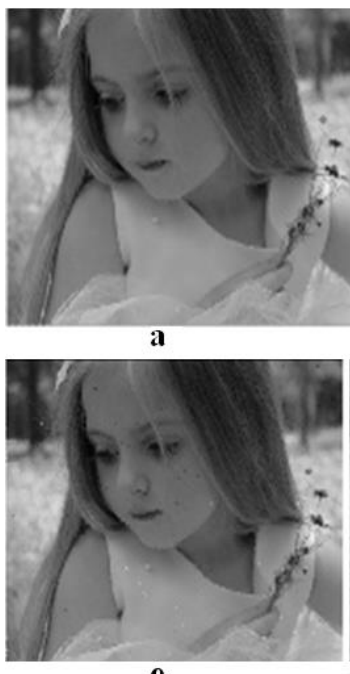

$\mathbf{e}$

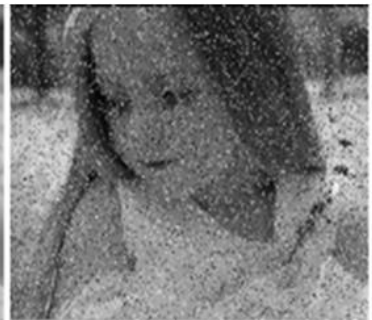

b

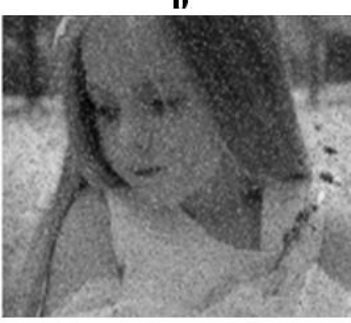

f

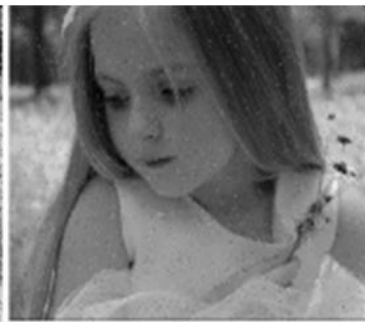

c

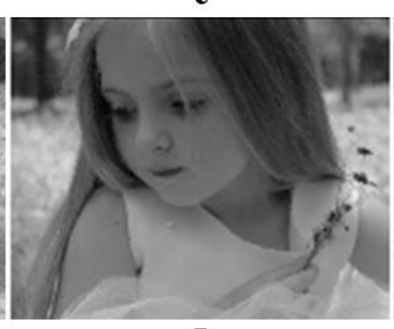

g
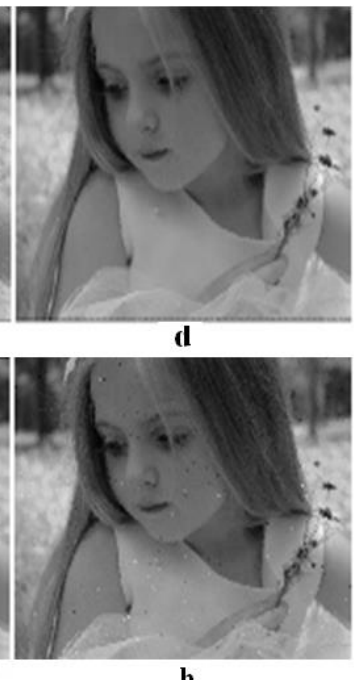

h

الثكل(9): نتائج المرشحات الجينية على صورة Girl والضوضاء نوع حبات الملح والففلل بنسبة 0.1. (a) الصورة الأصلية. (b) الصورة المشوهة. (c) الصورة المسترجعة بالمشح الجيني الأول. (d) الصورة المسترجعة بالمرشح الجيني

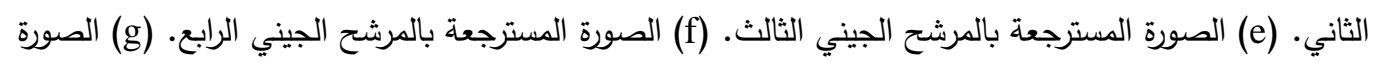
المسترجعة بالمرشح الجيني الخامس. (h) الصورة المسترجعة بالمرشح الجيني السادس. 


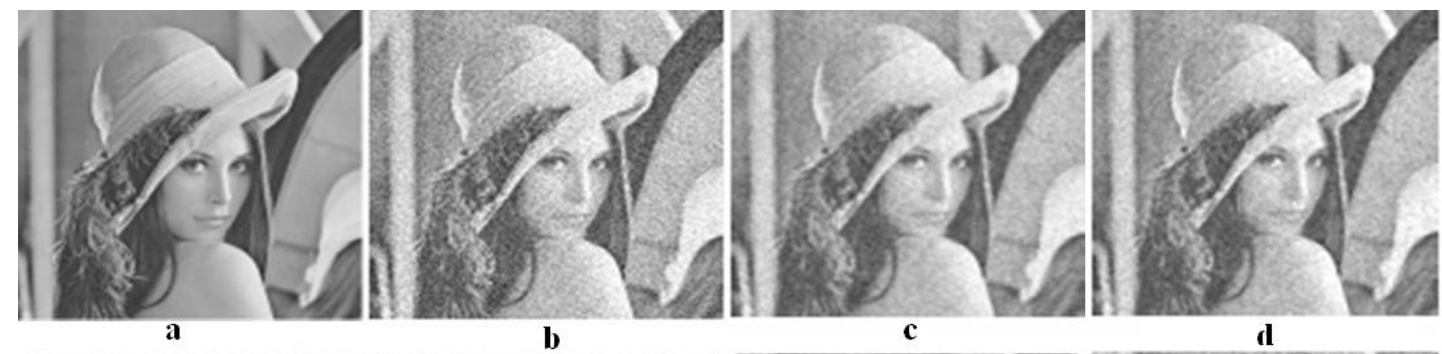

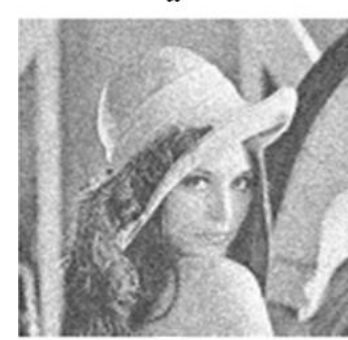

e

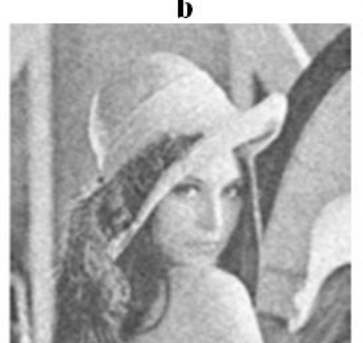

f

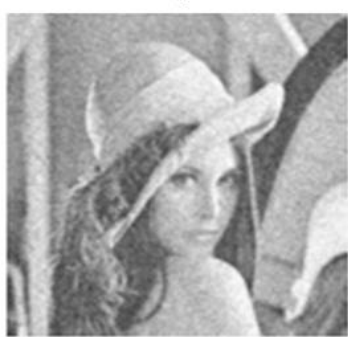

g

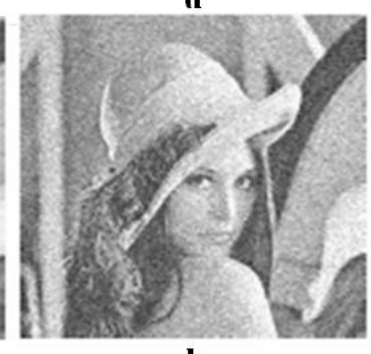

h

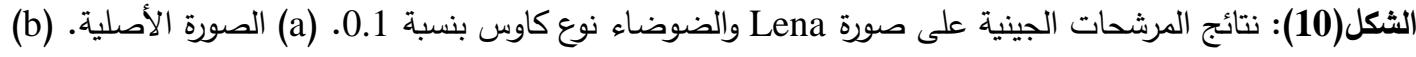

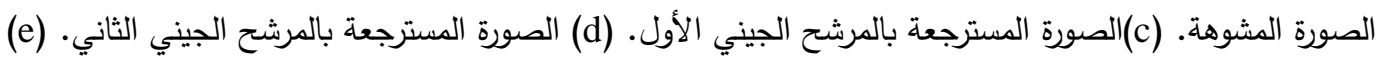

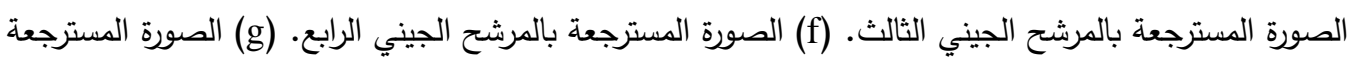
بالمرشح الجيني الخامس. (h) الصورة المسترجعة بالمرشح الجيني السادس.
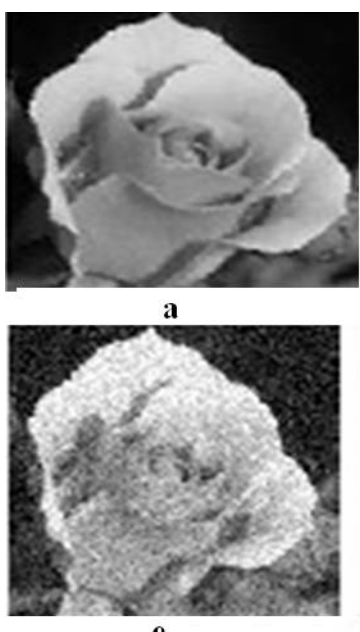

e

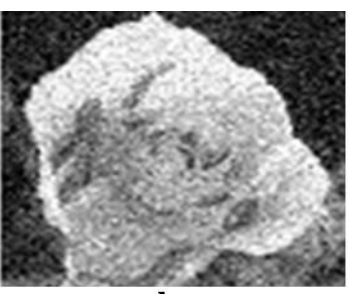

b

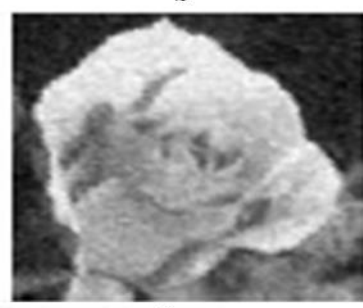

f
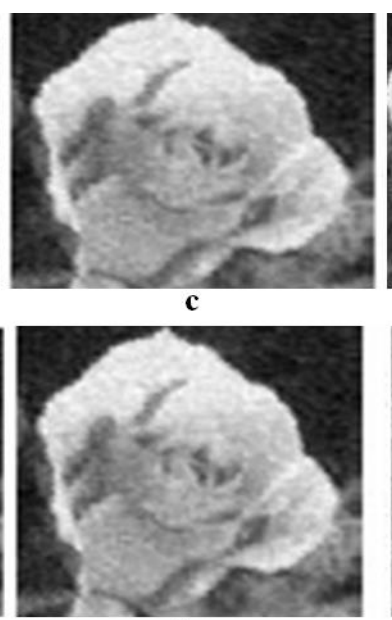

g
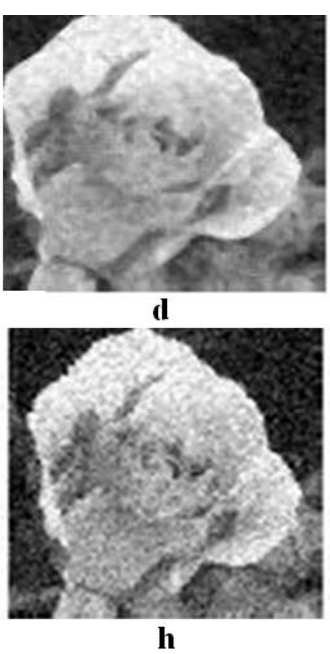

الثكل(11): نتائج المرشحات الجينية على صورة Flower والضوضاء نوع كاوس بنسبة 0.1. (a) الصورة الأصلية. (b) الصورة المشوهة. (c)(الصورة المسترجعة بالمرشح الجيني الأول. (d) الصورة المسترجعة بالمرشح الجيني الثاني.

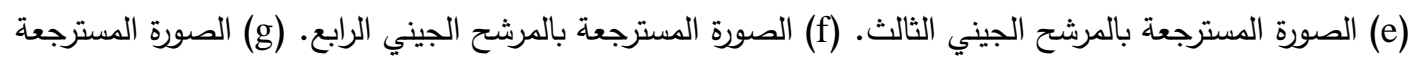
بالمرشح الجيني الخامس. (h) الصورة المسترجعة بالمرشح الجيني السادس. 


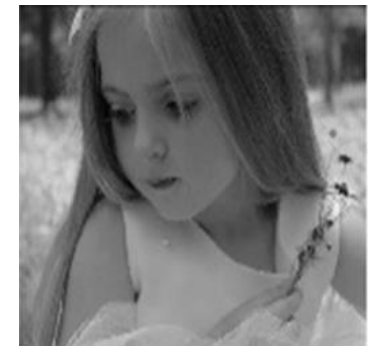

a

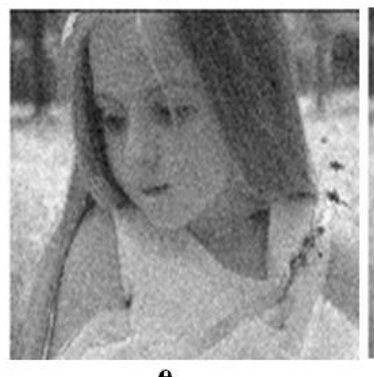

e

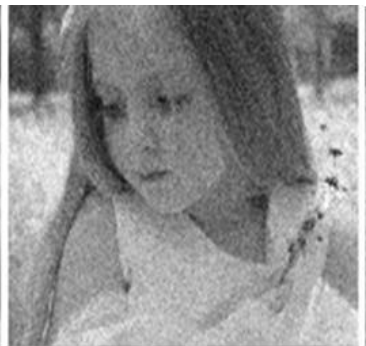

b

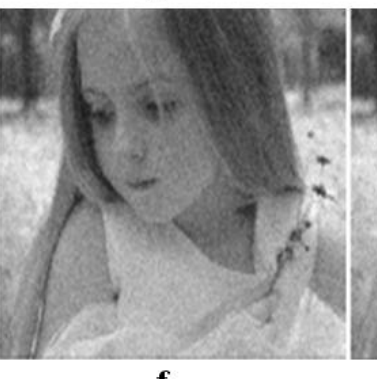

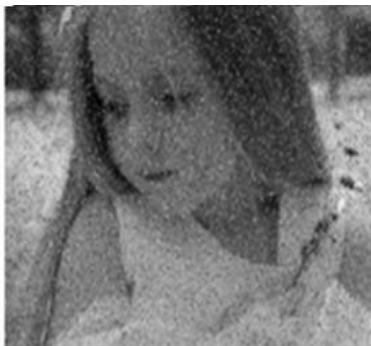

c

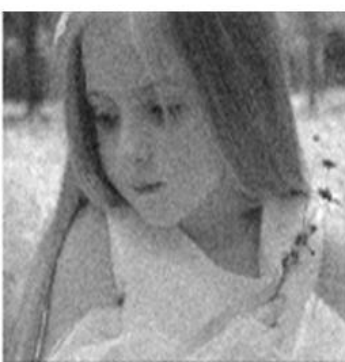

g
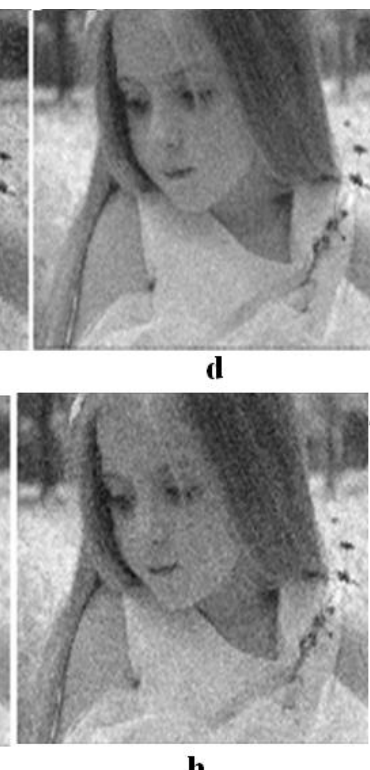

h

الشكل(12): نتائج المرشحات الجينية على صورة Girl والضوضاء نوع كاوس بنسبة 0.1. (a) الصورة الأصلية.(b)

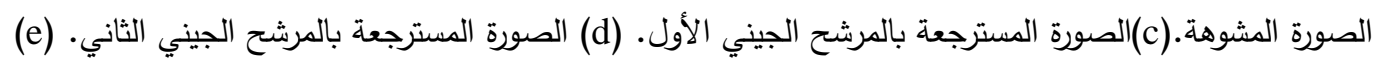

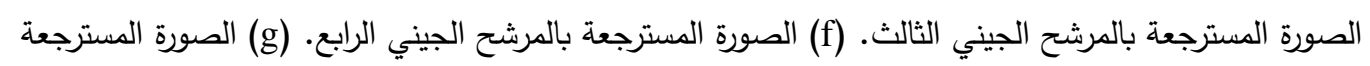
بالمرشح الجيني الخامس. (h)(الصورة المسترجعة بالمرشح الجيني السادس.

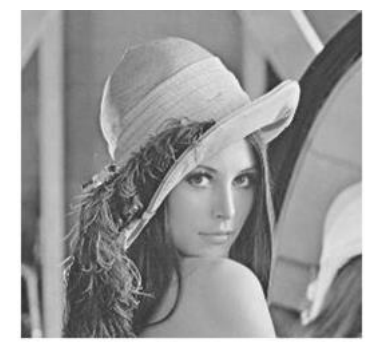

a

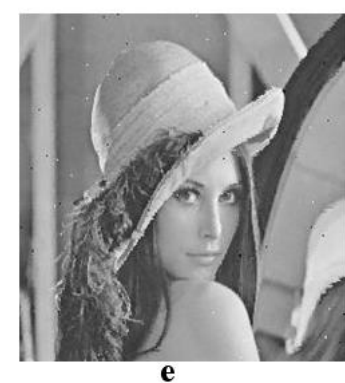

e

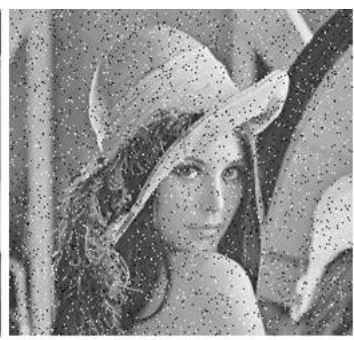

b

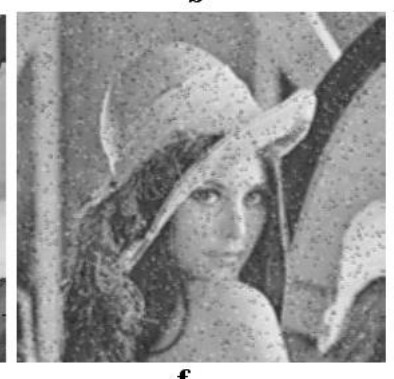

f

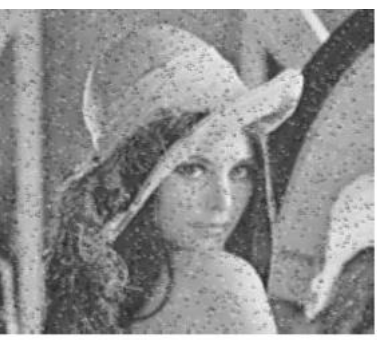

c

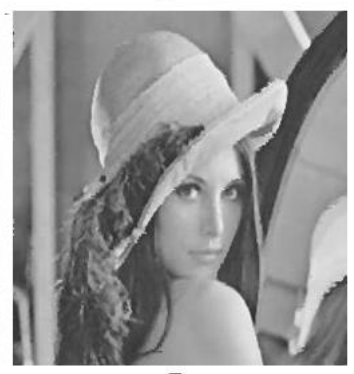

$\mathbf{g}$

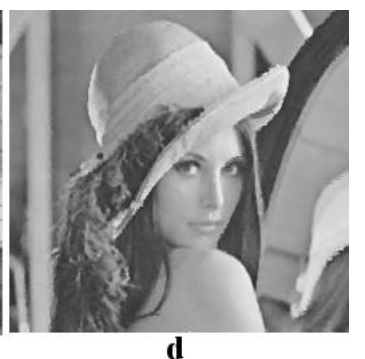

d

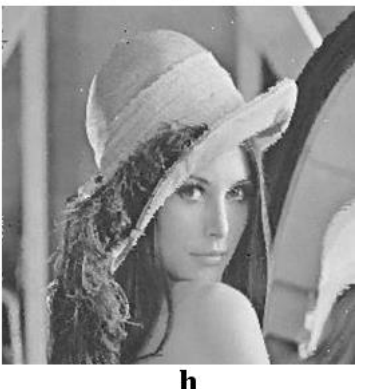

h

الثكل(13): نتائج المرشحات الجينية على صورة Lena والضوضاء نوع حبات الملح والفلفل بنسبة 0.05. (a) الصورة

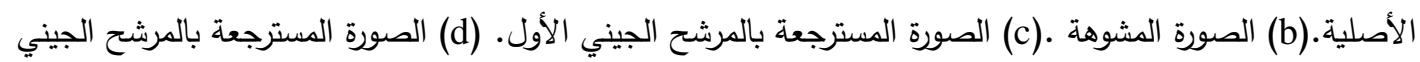

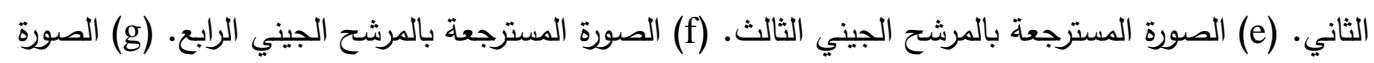
المسترجعة بالمرشح الجيني الخامس. (h) الصورة المسترجعة بالمرشح الجيني السادس. 

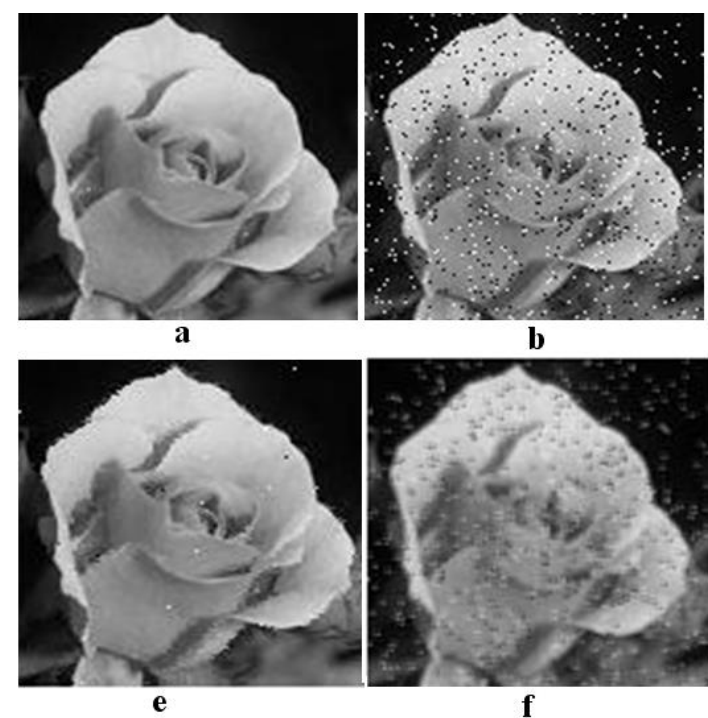

الثكل(14): نتائج المرشحات الجينية على صورة Flower والضوضاء نوع حبات الملح والفلفل بنسبة 0.05. (a) الصورة الأصلية.(b) الصورة المشوهة .(c) الصورة المسترجعة بالمرشح الجيني الأول. (d) الصورة المسترجعة بالمرشح

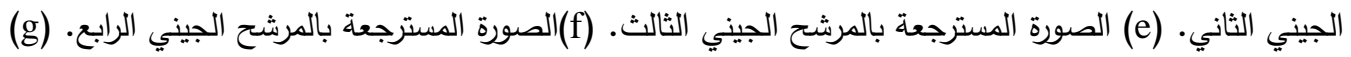
الصورة المسترجعة بالمرشح الجيني الخامس. (h) الصورة المسترجعة بالمرشح الجيني السادس.

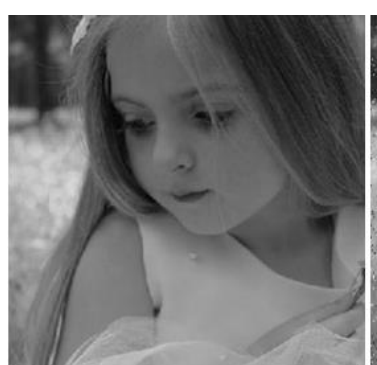

$\mathbf{a}$

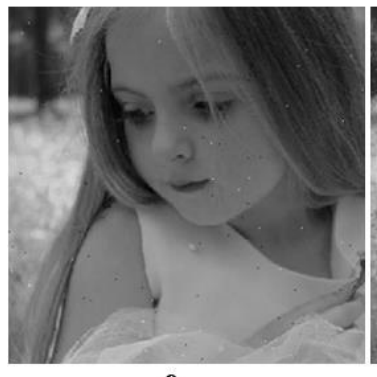

$\mathbf{e}$

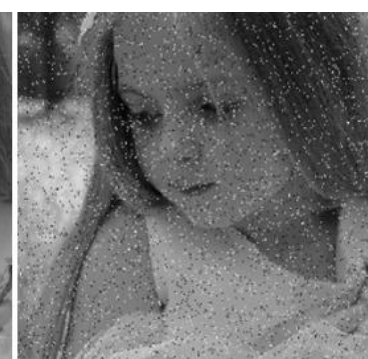

b

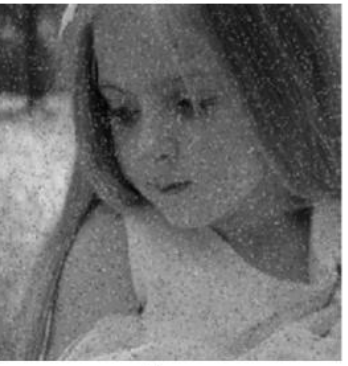

f

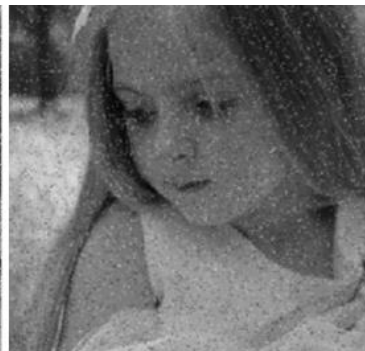

c

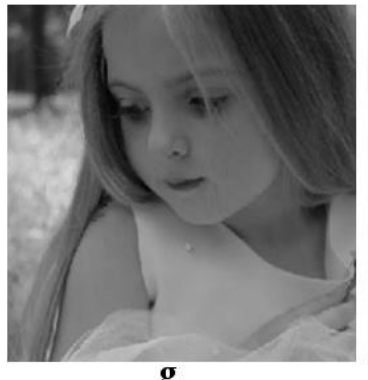

g
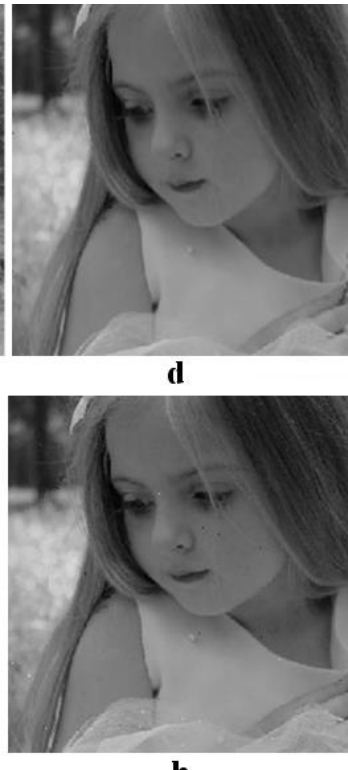

h

الثكل(15): نتائج المرشحات الجينية على صورة Girl والضوضاء نوع حبات الملح والفلفل بنسبة 0.05. (a) الصورة الصنا

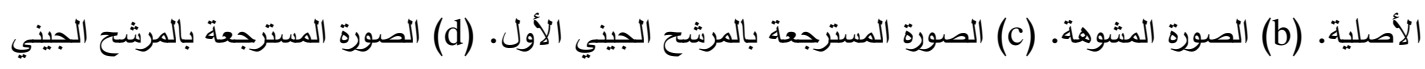

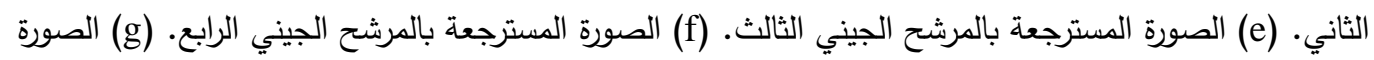
المسترجعة بالمرشح الجيني الخامس. (h) الصورة المسترجعة بالمرشح الجيني السادس. 

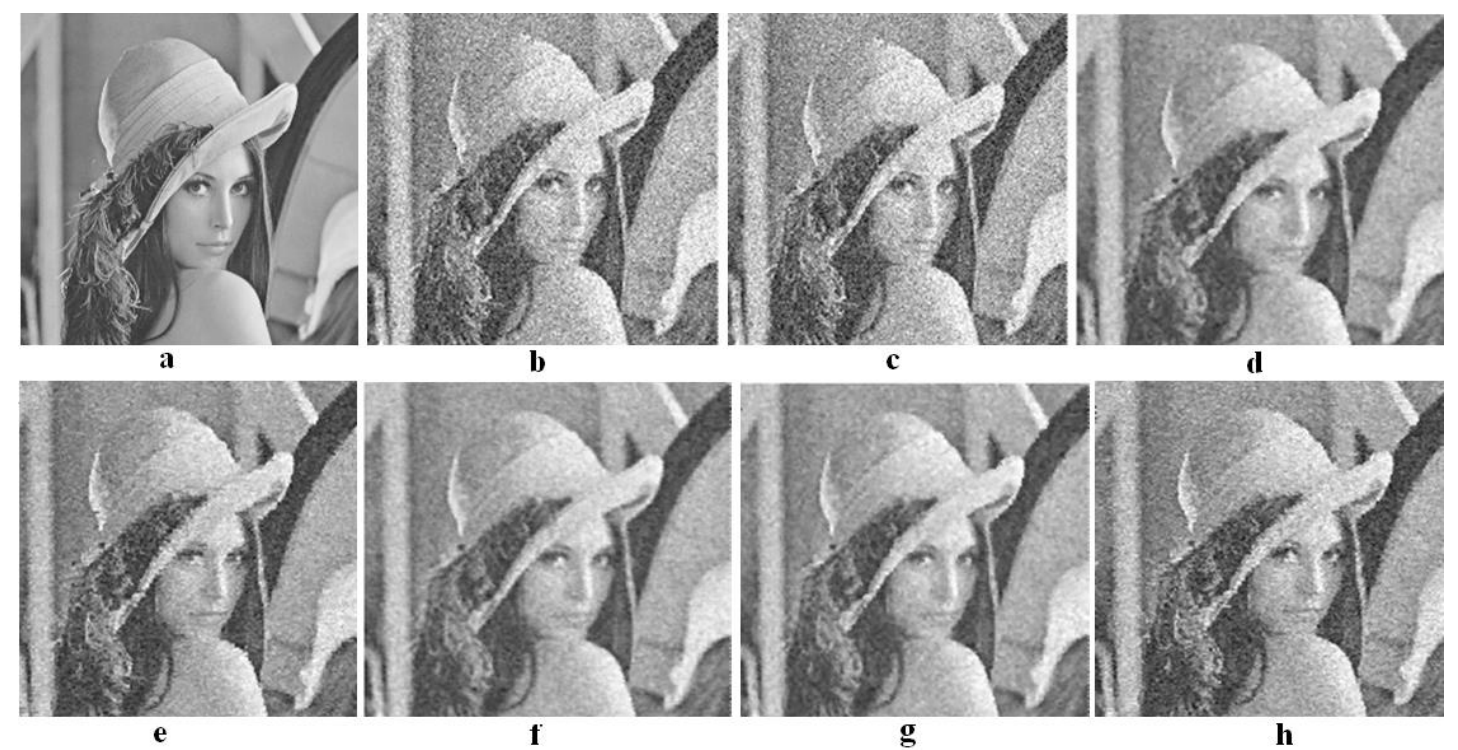

الثكل(16): نتائج المرشحات الجينية على صورة Lena والضوضاء نوع كاوس بنسبة 0.05. (a) الصورة الأصلية. (b) الصورة المشوهة. (c)(الصورة المسترجعة بالمرشح الجيني الأول. (d) الصورة المسترجعة بالمرشح الجيني الثاني.

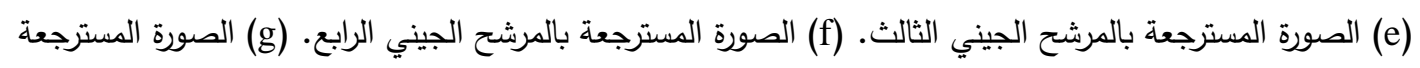

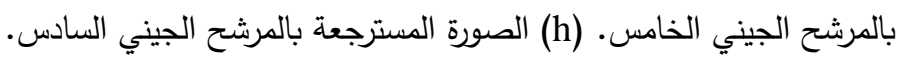
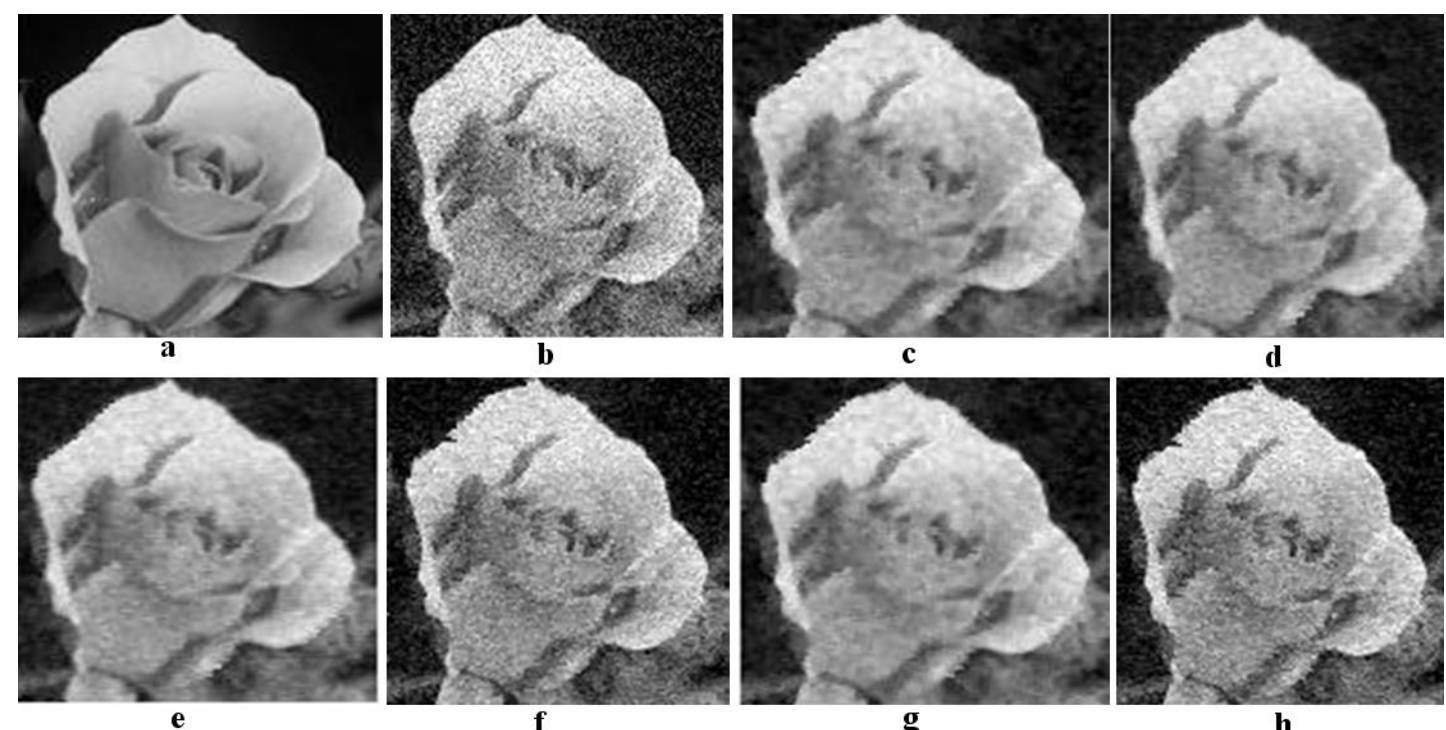

الثكل(17): نتائج المرشحات الجينية على صورة Flower والضوضاء نوع كاوس بنسبة 0.05. (a) الصورة الأصلية.

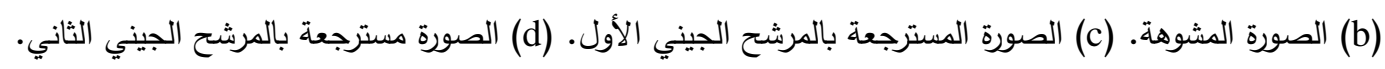

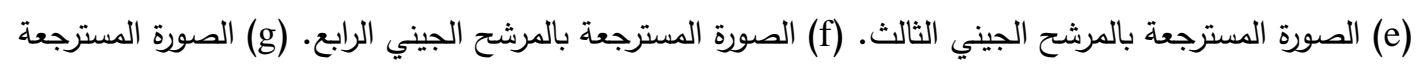
بالمرشح الجيني الخامس. (h) الصورة المسترجعة بالمرشح الجيني السادس. 


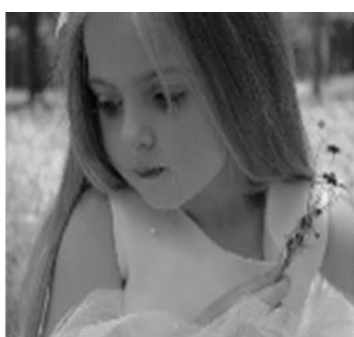

$\mathbf{a}$

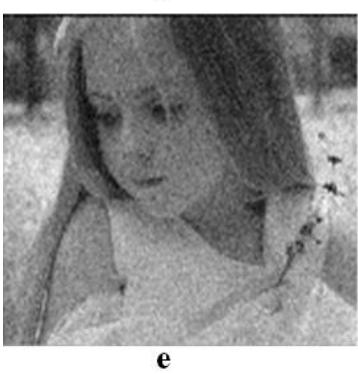

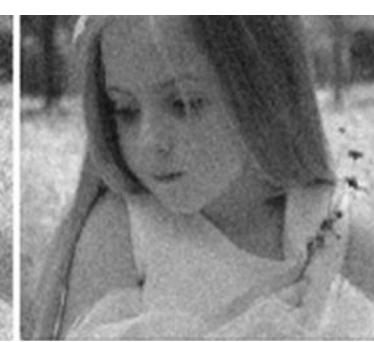

c

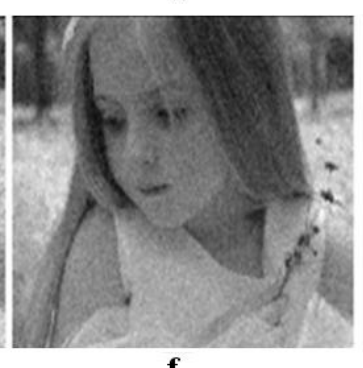

f

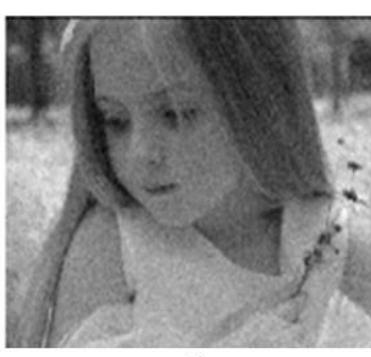

g
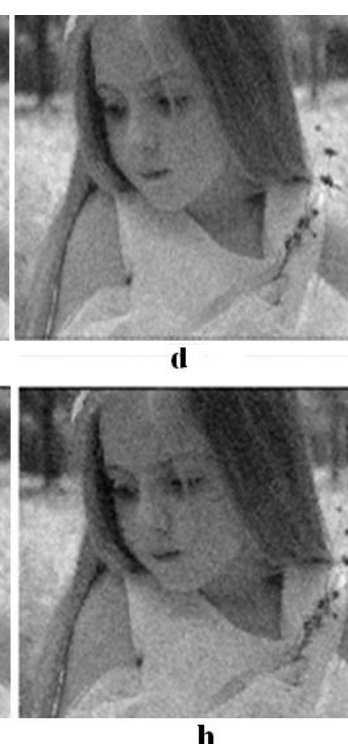

h

الثكل(18): نتائج المرشحات الجينية على صورة Girl والضوضاء نوع كاوس بنسبة 0.05. (a) الصورة الأصلية. (b)

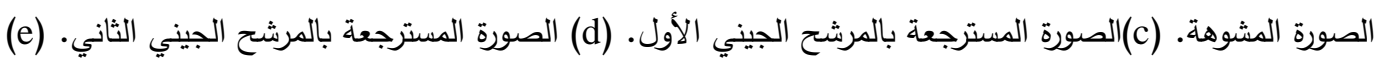

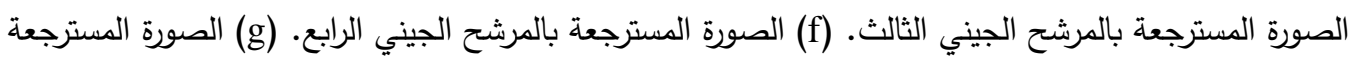
بالمرشح الجيني الخامس. (h) الصورة المسترجعة بالمرشح الجيني السادس.

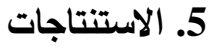

1. نلاحظ أن المرشح الجيني الخامس والمرشح الجيني الثاني أعطى أفضل النتائج مقارنـة بالمرشحات الأخرى من ناحية مقياس الرؤية البشرية المعتمد عليه بينما المرشح الجيني الأول والمرشح الجيني الرابع أعطيا نتائج

غير جيدة.

2. أظهر المرشح الجيني الثاني والخامس الأفضل لضوضـاء حبات الملح والفلفل لان مرشح الوسيط بالطرائق التقليدية يزيل ضوضـاء الملح والفلفل وبالرغم من جعله دالة هدف للخوارزمية الجينية فانه بقى نفس التأثير .

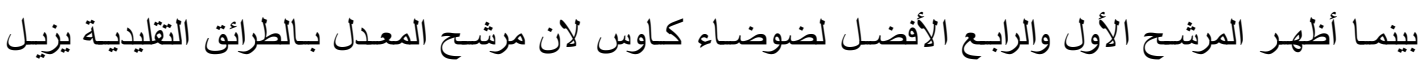

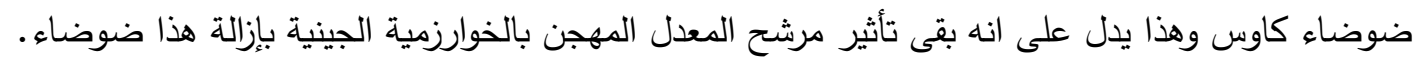

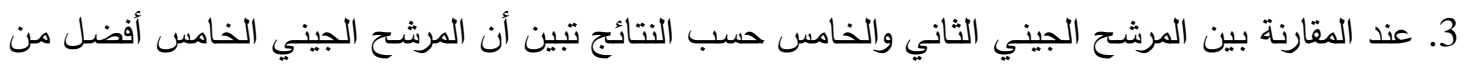

الثاني.

6

1. تطبيق أنواع أخرى من التداخل والطفرات على المرشحات الجينية المقترحة وإجراء المقارنة فيما بينها للتوصل

$$
\text { إلى نتائج أفضل. }
$$

2. تطبيق المرشحات الجينية المقترحة على صور متتوعة وصور ملونة.

3. تطوير المرشحات الاعتيادية وتهجينها مع الخوارزمية الجينية أو الثبكات العصبية أو المنطق المضبه ولهن. 


\section{المصادر}

[1] Davis, L. (1991), "Handbook of Genetic Algorithms", Von Nostrand Reinhold, New York.

[2] Fisher, R.; Perkin, S., Walker, A. and Wolfart, E.(2000), "Image Processing Learning Resources", HIPR2, Explor with JAVA.

[3] Glover, F. and Kochenberger, G.A. (2003), "Handbook of meta Heuristics", International series in operations research \& management science ISBN 1-40207263-5. Kluwer Academic Publishers.

[4] Goldberg, D.E., (2004), "Genetic Algorithms in Search, Optimization and Machine Learning", Pearson Education, Singapore .

[5] Gomes, J and Velho, L, "Image Processing for Computer Graphics", 1997, springer, New York.

[6] Gonzales, R.C. and Woods, R.E., (2002), "Digital Image processing", Second Edition, Prentice Hall, New Jersey.

[7] Gonzalez, R.C. and Woods, R.E., (2004), "Digital Image Processing Using MATLAB7", Person Education Asia.

[8] Gupta, G., (2011), "Algorithm for Image Processing Using Improved Median Filter and Comparison of Mean, Median and Improved Median Filter", International Journal of Soft Computing and Engineering (IJSCE) ISSN: 22312307, Volume-1, Issue-5, page 304-311.

[9] How-Lung, E. and Kai-Kuang M., (2001), "Noise Adaptive Soft-Switching Median Filter", IEEE Transactions On Image Processing, Vol. 10, No. 2, pp. 242- 251

[10] Jain, A.K. (1982), "Fundamentals of Digital Image Processing", Prentice-Hall Inc.

[11] Jansen, M. and Bultheel, A., (1999), "Multiple wavelet threshold estimation by generalized cross validation for images with correlated noise," IEEE Tarns. on Image Processing, Vol. 8, No. 7, pp. 947-953 .

[12] Jansen, M., Malfait, M. and Bultheel, A. (1997), "Generalized cross validation for wavelet thresholding", Signal Processing, Vol. 56, pp. 33-34.

[13] Jensen J. R.(1986), “Introductory Digital image processing", A Remote sensing Perspective, Prentice Hall.

[14] Lutton, E. and Martinez, P., (1994), "A Genetic Algorithm for the Detection of 2D Geometric Primitives in Images", Proceedings of the 12th International Conference on Pattern Recognition, Jerusalem, Israel, 9-13 October, Vol. 1, pp. 526-528.

[15] Mainzer, T., (2002), "Genetic Algorithm for Shape Detection", Technical Report no. DCSE/TR-2002-06, University of West Bohemia Pearson Eductiaon, Singapore. 
[16] Paulinas, M. and Ušinskas, A., (2007), "A Survey Of Genetic Algorithms Applications For Image Enhancement And Segmentation", ISSN 1392-124X Information Technology And Control, 2007, Vol.36, No.3, pp.278-284.

[17] Phillips, D., (2000), "Image Processing in C", Second Edition, CMP Media, Inc.

[18] Qiu, G., (1996), "An Improved Recursive Median Filtering Scheme for Image Processing" , IEEE Transactions On Image Processing, VOL. 5, NO. 4, pp. 646648.

[19] Ravi Kumar, Ch and Srivathsa, S.K., (2009), "EHW Architecture for Design of Adaptive Median Filter for Noise Reduction", European Journal of Scientific Research ISSN 1450-216X, Vol.36, No.3, pp. 473-479.

[20] Russo F.,( 2002), "An image enhancement technique combining sharpening and noise reduction," IEEE Trans. Instrum.Meas., vol. 51, no. 4, pp. 824-828.

[21] Santa-Cruza, D., Ebrahimia, T., Askelöfb J., Larssonb, M.and Christopoulosb C. A., (2000),"JPEG 2000 still image coding versus other standards", In Proc. of the SPIE's 45th annual meeting, Applications of Digital Image Processing XXIII, volume 4115, pages 446-454.

[22] Sovilj-Nikic,S. and Sovilj-Nikic,I.,( 2007)," Application of Genetic Algorithm in Median Filtering", Proceedings of the International Multiconference on Computer Science and Information Technology ISSN 1896-7094 pp. 127 - 139.

[23] Umbaugh S.E.,"Computer Vision and Image Processing ",2006,Prentice Hall PTR,USA.

[24] Vainio,O., Neuvo,Y. and Steven E. B., (1989),“A Signal Processor for MedianBased Algorithms",IEEE Transactions on Acoustics, Speech, Processing VOL 37. NO. 9, September.

[25] Van De Ville, D.,Nachtegael,M, Van der Weken D., Philips,W. and Lemahieu,I (2002) " Noise Reduction by Fuzzy Image Filtering" , IEEE Transactions On Fuzzy Systems, VOL. 11, NO. 4,page 429-436.

[26] Wang and Vagnercci, A.H.(1981), “ Digital image Enhancement”, IEEE Transactions on Computer, Vision, Graphics image processing, vol-24.pp363381. 